\title{
Imaging of hip and thigh muscle injury: a pictorial review
}

\author{
Kolja M. Thierfelder ${ }^{1 *}$, Judith S. Gerhardt ${ }^{1}$, Ioan N. Gemescu ${ }^{2}$, Susan Notohamiprodjo ${ }^{3}$, Christoph Rehnitz ${ }^{4}$ and \\ Marc-André Weber ${ }^{1}$
}

\begin{abstract}
Muscle injuries of the hip and thigh are a highly relevant issue in competitive sports imaging. The gold standard in diagnostic imaging of muscle injuries is magnetic resonance imaging (MRI). Radiologists need to be familiar with typical MRI findings in order to accurately detect and classify muscle injuries. Proper interpretation of the findings is crucial, especially in elite athletes. In soccer players, muscle injuries of the hip and thigh are the most common reason for missing a game.

The present pictorial review deals with the diagnostic assessment, especially MRI, of muscle injuries of the hip and thigh. Typical MR findings in muscle injuries include edema, hematoma, and tendinous avulsion as well as partial or complete muscle tear. To estimate the time to return to play, a grading into three groups-muscle strain, partial tear, complete tear-has traditionally been used. Taking into account the most recent literature, there are other prognostic factors such as the longitudinal length of a tear, the tendon's intramuscular component, or persisting edema.
\end{abstract}

Keywords: Muscle injury, Muscle tear, Magnetic resonance imaging, Soccer, Hip, Thigh

\section{Introduction}

Diagnosing and grading of muscle injuries are highly relevant issues in professional sports [1]. During training and matches, the musculoskeletal system of soccer players is exposed to complex biomechanical forces [2, 3]. As a result of muscle injuries, $37 \%$ of professional soccer players miss training or matches each season $[4,5]$. In over $90 \%$ of cases, the muscles of the lower extremities are affected $[5,6]$. Muscle injuries are not specific to certain sports, but there are some typical biomechanics and some injury patterns that occur more often in particular sporting activities (Table 1) $[7,8]$. Among soccer players, about $35 \%$ of injuries are muscle injuries, which often occur during a match $[5,9]$. In soccer, muscle injuries are the most frequent cause for missing a game $[9,10]$.

In the vast majority (around 96\%) of muscle injuries, there is an indirect mechanism of accident, often with the result of a muscle tear [4]. In around $2 \%$, a direct trauma is the reason for a muscle injury. In contrast to

\footnotetext{
* Correspondence: kolja.thierfelder@med.uni-rostock.de

${ }^{1}$ Institute of Diagnostic and Interventional Radiology, Pediatric Radiology and Neuroradiology, University Medical Center Rostock, Ernst-Heydemann-Str. 6, 18057 Rostock, Germany

Full list of author information is available at the end of the article
}

indirect injuries, there generally is a muscle contusion after direct trauma [11]. A blunt direct trauma that can induce a bleeding deep within the muscle, resulting in a hematoma, which is mostly clearly detectable by MRI (Fig. 1) [4]. Muscle contusions generally heal relatively fast, making return to play often possible within a week. In rare cases, direct muscle contusions can result in compartment syndrome $[4,9]$.

Unlike muscle contusions, muscle tears are generally caused not by a direct but an indirect injury. This indirect trauma is induced by eccentric contraction [12]. In MRI, typical features of indirect muscle injuries are hematoma, fiber disruption, and muscle edema. The muscles with a high risk for tears are types with a high proportion of type II fibers, muscles with multiple heads, and muscles extending over two joints like the M. biceps femoris, M. rectus femoris, and the adductor muscles [13]. There is also a higher risk for muscles that show high peak levels of stress in specific movements, e.g., the M. rectus femoris (during shooting) or the M. biceps femoris (during sprinting) [5]. In soccer players, the most commonly involved muscles are the hamstrings $(37 \%)$, adductors $(23 \%)$, and the quadriceps muscle (19\%) [5]. 
Table 1 Typical injuries for the most common sports [8]

\begin{tabular}{|c|c|}
\hline Sport & Most common injuries \\
\hline Soccer & Hamstring and quadriceps strain/cramps/contusions \\
\hline Tennis & $\begin{array}{l}\text { Rotator cuff tears, lateral epiconodylitis } \\
\text { ("tennis elbow") }\end{array}$ \\
\hline Football & $\begin{array}{l}\text { Lateral ankle sprain, ACL tear, ACC joint separation, } \\
\text { hamstring strains }\end{array}$ \\
\hline Basketball & Ankle sprains, ankle/foot fractures, knee joint injuries \\
\hline Baseball & $\begin{array}{l}\text { Rotator cuff tears, SLAP lesions, posteromedial elbow } \\
\text { impingement }\end{array}$ \\
\hline Volleyball & $\begin{array}{l}\text { Acute ankle sprain, chronic/overuse knee injuries, } \\
\text { shoulder overuse injuries }\end{array}$ \\
\hline Rugby & Thoracic cage fractures, lower extremity muscle strains \\
\hline Cycling & $\begin{array}{l}\text { Acute trauma targeting any region (falling or traffic } \\
\text { accident), anterior knee injury }\end{array}$ \\
\hline Skiing & $\begin{array}{l}\text { ACL/MCL sprains, femoroacetabular impingement, } \\
\text { thumb ulnar collateral ligament sprain (skier's thumb) }\end{array}$ \\
\hline
\end{tabular}

$A C L$ anterior cruciate ligament, $A C C$ acromioclavicular, SLAP superior labral tear from anterior to posterior, $M C L$ medial collateral ligament

The localization of a muscle tear depends on the age of the patient. In young athletes, the muscle typically tears at the non-fused apophysis. In elder people, the tear is most commonly located at the tendon, which is often already affected by degenerative changes. In adults of intermediate age, the location of the tear is often the myotendinous junction, which is the weakest link in the muscle-tendon-bone chain $[7,12]$.

From a radiological point of view, it is important to know the typical imaging findings and to be able to differentiate among the different kinds of muscle injuries. The prognosis varies widely among the different types, and there are many different therapy options.

\section{Imaging techniques}

In muscle injuries of the hip and thigh, there is a large variety of imaging patterns, ranging from very subtle changes in muscle strains to complete tears with retraction of the muscle and large hematoma. Both ultrasound (US) and MRI are generally suitable to evaluate muscle injuries $[14,15]$. US is often used as the first modality as it is widely available-even in proximity to the sports arena of several professional soccer clubs, fast, easy to use, and affordable. However, US is known to be highly user-dependent. In addition, the determination of the length of a muscle tear is difficult and small hematomas can be missed, especially within the first $24 \mathrm{~h}$ after injury. MRI is a more elaborate imaging technique that is considered the reference standard for muscle injuries. It allows for an excellent depiction of the most relevant findings in muscle injuries [6] and has a high sensitivity in identifying acute and chronic soft tissue alteration [16].

To assess muscle injuries on MRI, fluid-sensitive short-tau inversion recovery (STIR) or proton density fat-saturated (PDfs) sequences on the one hand and T1-weighted sequences on the other hand should be acquired as a minimum. Fluid-sensitive sequences allow to visualize edema, muscle tear, hematoma, and bone bruise. While PDf sequences provide more anatomical information, STIR is less sensitive to metal artifacts and patient movement. On the other hand, PD sequences have a higher signal to noise ratio (SNR) compared to STIR sequences. T1-weighted sequences allow for an assessment of (stress) fractures including bony avulsions and hematoma. Optionally, T2-weighted sequences can be used. Contrast-enhanced sequences are not necessary after acute trauma. Furthermore, it is a common practice to assess anatomical features on

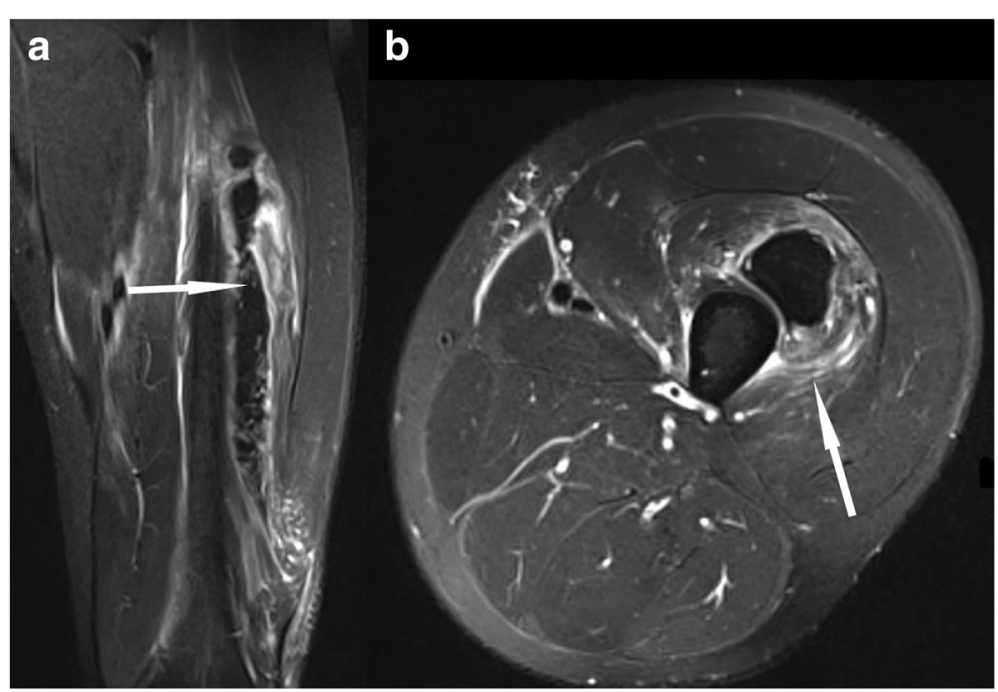

Fig. 1 Coronal (a) and axial STIR (b). Feathery edema around hematoma within the M. vastus intermedius after blunt direct trauma 
non-fat-suppressed images. Dixon sequences (chemical-shift imaging) can be useful as they provide separated water-only and fat-only images. This technique can provide a more uniform fat suppression $[17,18]$.

\section{Grading and prognosis}

Muscle injuries in athletes are quite diverse and challenging to precisely define [4]. So far, a clear consensus on a clinical grading system does not exist. Every grading system has its strengths and weaknesses. The term muscle strain, though it is not consistently interpreted, is often used [4]. In order to determine the most suitable therapy and to estimate the time to return to play, a clinical examination is performed. It is generally based on a grading into three levels, considering bulge and loss of strength. One of the most commonly used clinical grading systems was introduced by O'Donoghue in 1962. Since then, several other muscle injury classifications were proposed, as presented in Table 2 [4].

The most common MRI-based grading system of muscle injuries, modified by the ultrasound-based Peetrons classification, distinguishes four grades [3] (Table 3). It provides only a rough estimate, and it suffers from the drawback of the wide range of grade 2 injuries and the non-existence of a rating for the tendon's intramuscular component. Grade 0 is used in cases with a normal MRI. Grade 1 includes all cases with an MRI showing edema, but no structural damage or hemorrhage [4] (Fig. 2). Grade 2 is a partial and grade 3 a complete tear of the muscle (Fig. 3). The time to return to play is closely correlated to the grade-the higher the grade, the longer the time to return to play $[3,4]$. The MRI correlate of a grade 1 muscle strain is an intramuscular hyperintensity on fluid-sensitive sequences without evidence of muscle fiber disruption (Fig. 2). The wide range of grade 2 injuries comprises tiny fiber discontinuities (Fig. 3) to large subtotal muscle ruptures (Fig. 4). A grade 3 injury represents a complete muscle tear (Fig. 5). In grade 3 injuries, the extent of muscle retraction needs to be determined and reported. Grade 3 injuries may require surgery.

Further predictive factors for the lay-off time are the longitudinal length as well as proportion and volume of

Table 2 Clinical grading systems for muscle injuries

\begin{tabular}{llll}
\hline $\begin{array}{l}\text { Name of } \\
\text { Classification }\end{array}$ & Year & Focus (if any) & $\begin{array}{l}\text { Number of } \\
\text { grades used }\end{array}$ \\
\hline O'Donoghue & 1962 & - & 3 \\
Ryan & 1969 & Quadriceps (initially) & 4 \\
Takebayashi & 1995 & Ultrasound & 3 \\
Peetrons & 2002 & Ultrasound & 3 \\
Stoller & 2007 & MRI & 3 \\
Munich & 2012 & MRI & $4+$ contusions \\
\hline
\end{tabular}

Table 3 MRI-based classification system of muscle injuries and resulting lay-off time $[3,4]$

\begin{tabular}{lll}
\hline Grade & MRl finding & $\begin{array}{l}\text { Lay-off } \\
\text { time (days) }\end{array}$ \\
\hline 0 & Normal MRI & $8 \pm 3$ \\
1 & Edema (Fig. 2) & $17 \pm 10$ \\
2 & Partial tear & $22 \pm 11$ \\
3 & (Figs. 3 and 4) & $73 \pm 60$ \\
\hline
\end{tabular}

the muscle injury on MRI $[6,19]$. The longitudinal length of the muscle injury, although it is not considered in the common grading system presented in Table 3, has the highest prognostic value. It reflects the number of muscle units that are separated from the aponeurosis [19]. The injury of the intramuscular component of the tendon (Fig. 6), which is not considered in the traditional grading system, is also associated with a delayed return to play [7, 20]. Moreover, a previous injury of a high-risk muscle increases the risk of another injury of the same muscle [21]. In elite soccer players, $16 \%$ of muscle injuries are re-injuries, which imply a 30\% longer lay-off time [4]. An underestimated injury and the subsequent premature return to play is a common reason for recurrent muscle injuries. Also, an inadequate rehabilitation is a risk factor for muscle injuries in soccer players [9].

The common grading systems do not further divide the grades, so that injuries with a diverse etiology and prognosis are grouped together, especially in the heterogeneous group of partial tears. The Munich muscle injury classification is a comprehensive grading system for athletic muscle injuries with the aim of grading the injuries in a more comprehensive and comparable way by standardizing terms of muscle injuries. Indirect muscle injuries are classified into functional and structural disorders with sub-classification: functional disorders are defined as acute indirect muscle disorders without macroscopic evidence (in MRI or ultrasound) of muscular tear while structural disorders refer to any acute indirect muscle injury with macroscopic evidence (in MRI or ultrasound) of muscle tear [4]. Compared to functional muscle disorders, structural injuries are associated with longer time to return to play. This simple classification provides a good evaluation of injuries and helps to provide a proper diagnosis while reducing the chances of miscommunication and the rate of recurrence/complications. It has a strong correlation with the time to return to play [4].

\section{Differential diagnoses of muscle injuries of the hip and thigh}

Differential diagnoses in MRI of muscle injuries are exercise-related delayed onset muscle soreness (DOMS) 


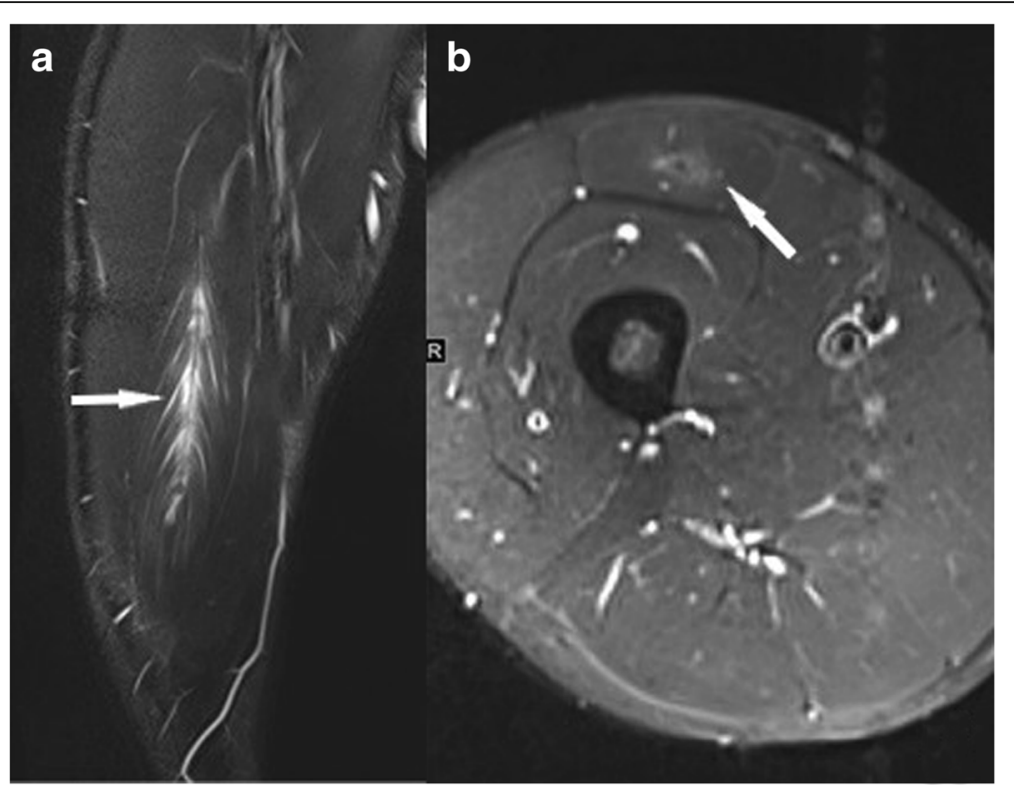

Fig. 2 Coronal (a) and axial STIR (b). Grade 1 muscle injury of the M. rectus femoris with feathery intramuscular edema, but without muscle fiber discontinuity

and post-exercise edema. DOMS occurs 1 or 2 days after exertion and manifests as local pain, soreness, and soft tissue swelling, often increasing over the course of 1 week (Fig. 7). The muscle appearance is similar to a grade 1 strain injury and shows a feathery-like pattern on fluidsensitive sequences in relation to an inter-fascicular edema

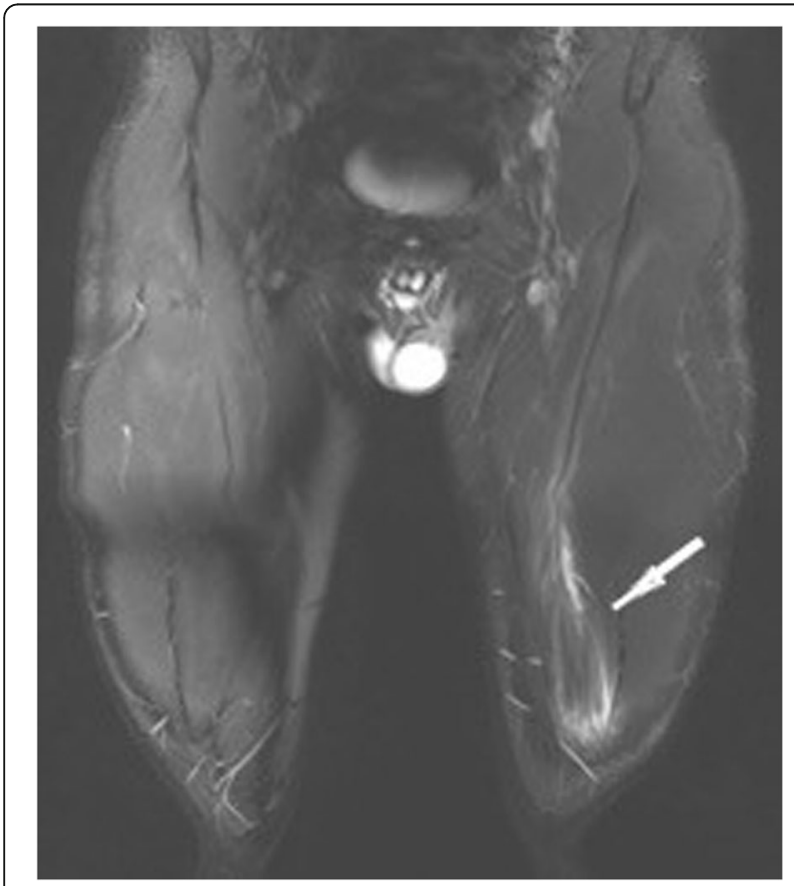

Fig. 3 Coronal STIR sequence. Mild grade 2 injury with a partial tear of the $\mathrm{M}$. rectus femoris with edema and hematoma

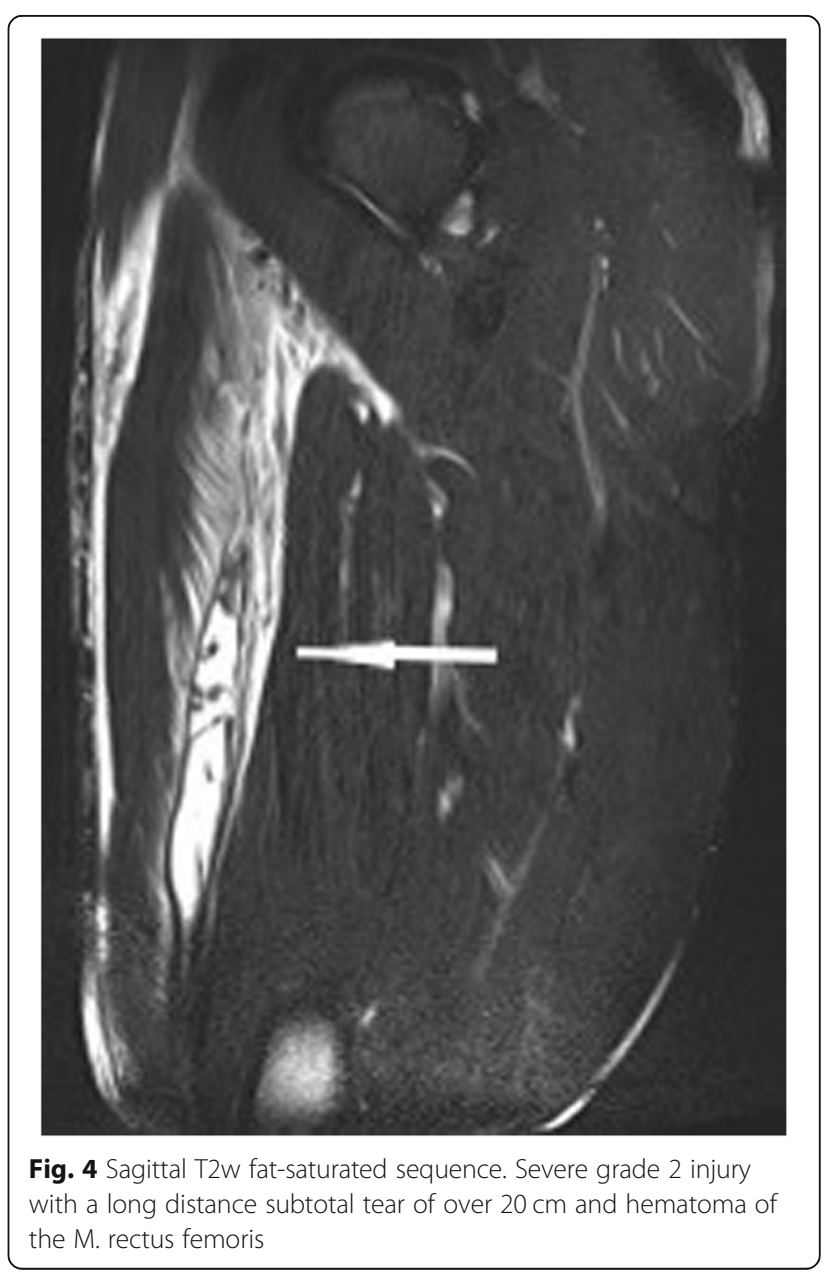




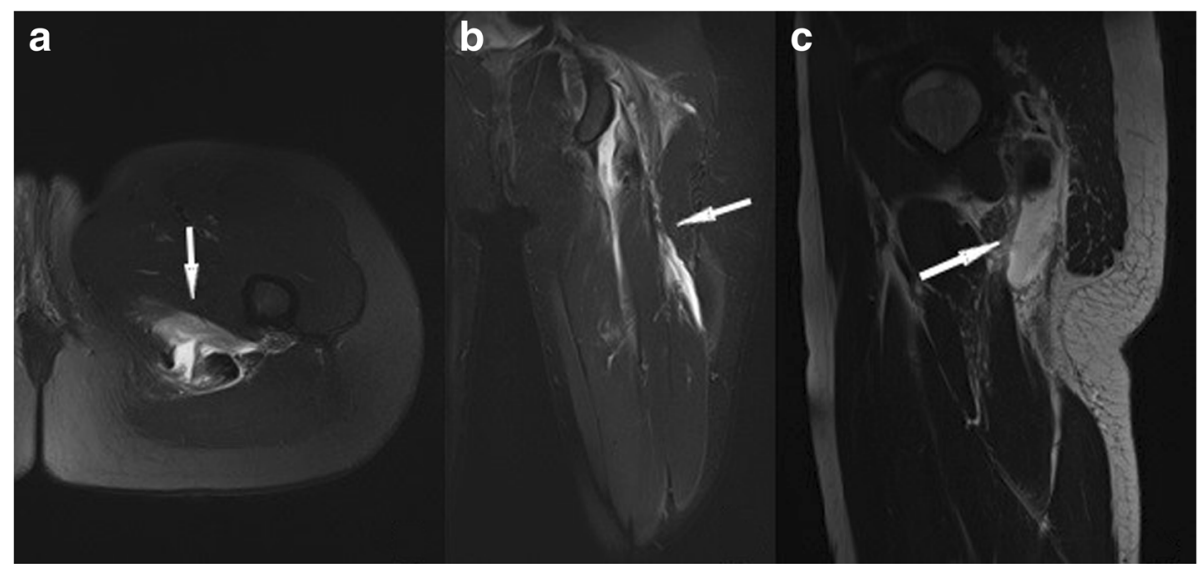

Fig. 5 Axial PDfs (a), coronal PDfs (b), and sagittal T2w (c) images of a grade 3 injury of the hamstrings

[22]. Unlike DOMS, the post-exercise edema usually resolves within few hours after exercise. As shown in Fig. 8, it can also appear similar to a grade 1 tear. Also, the IVIM sequence which will be mentioned below provides information on microvascular perfusion after muscle effort [23].

\section{Complications}

Possible complications of muscle injuries are muscle hernia, acute or chronic exertional compartment syndrome, myositis ossificans, calcific myonecrosis, and Morel-Lavallée lesions [24]. Muscle hernias manifest in herniation of the muscle tissue through a small fascial defect in relation to a prior blunt or penetrating muscle trauma (Fig. 9). Muscle hernias increase in size during activity and can sometimes be indetectable during rest periods, thus making dynamic or standing ultrasound a requirement [25-28].

A compartment syndrome is caused by muscle anoxia by an increasing pressure within the compartment. The chronic exertional compartment syndrome results from an increased compartment pressure during exercise and resolves with rest. An acute compartment syndrome, on the other hand, is a surgical emergency presenting in with pain, which is disproportionate to the injury. On MRI, there are often bilateral signal hyperintensity peaks at exercise with delayed return to normal signal (Fig. 10).

Myositis ossificans (better: heterotopic ossification, as the condition is not an inflammatory process) is a common sequelae of muscle injuries. Initially, hematoma and edema are present. On MRI, there is a high signal intensity on fluid-sensitive images and an increased enhancement after contrast administration. After about 6 weeks, typical imaging features with a peripheral rim of ossification that progresses towards the center can be found [25] (Fig. 11).

Morel-Lavallée lesions result from a closed degloving injury as a result of shear forces associated with a serve trauma and resulting separation of the subcutaneous tissue from fascia [29]. On MRI, it is often accompanied with recurrent fluid accumulation, while the fluid is of variable signal intensity (Fig. 12).

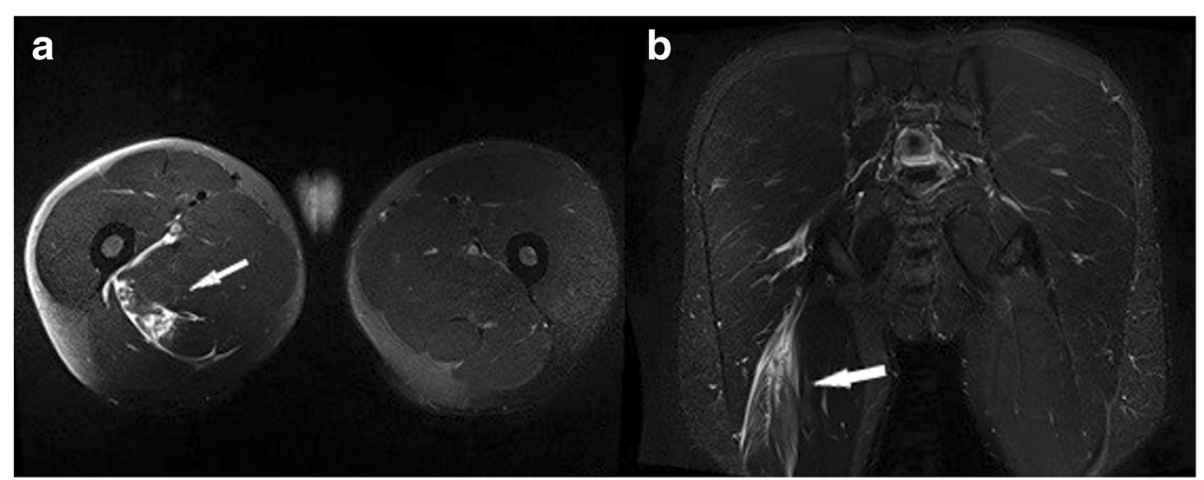

Fig. 6 Axial T2 fs (a) and coronal STIR image (b) showing tears of the intramuscular component of the adductor and semitendinosus tendon 


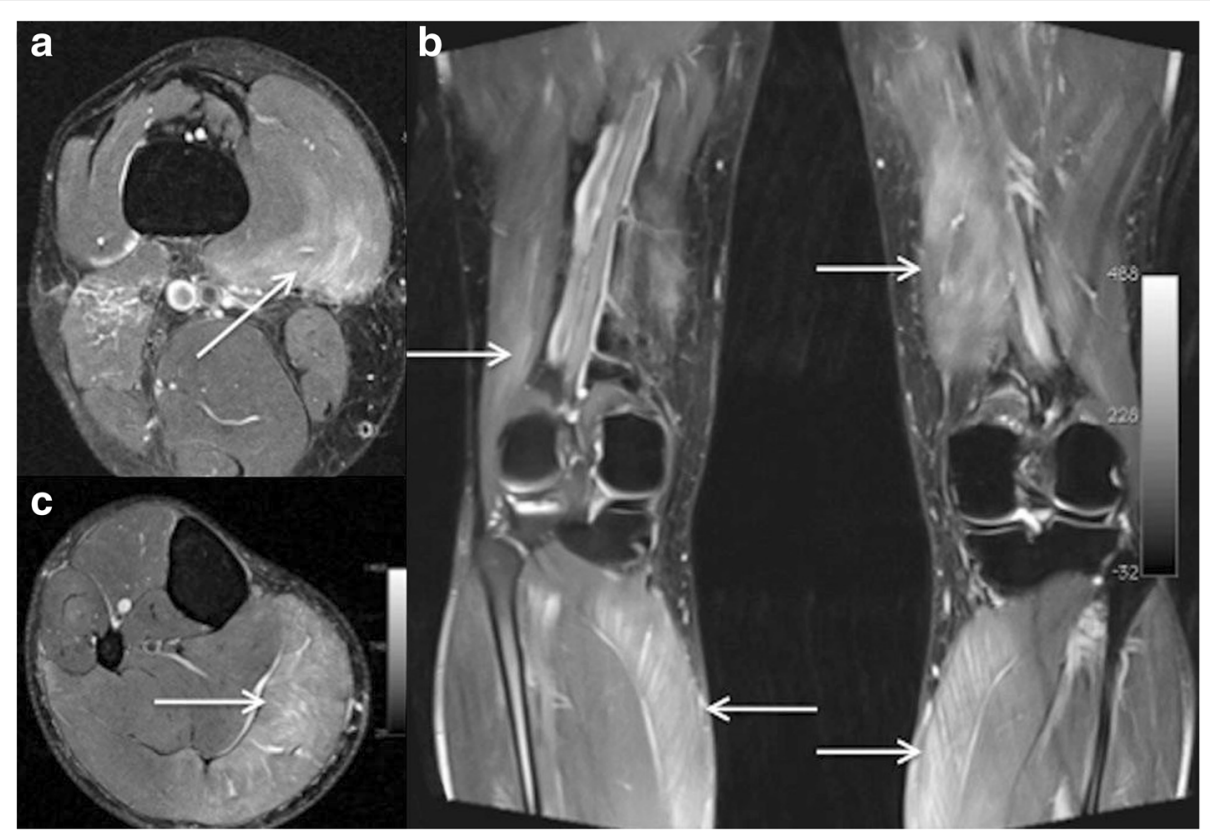

Fig. 7 Axial $(\mathbf{a}, \mathbf{c})$ and coronal (b) PDf-weighted MR images in an athlete with delayed onset muscle soreness 4 days after completing a triathlon. There is an extensive lower limb edema bilaterally, predominately involving the vastus medialis and the medial head of gastrocnemius (arrows) (images with courtesy of Dr. Andrew Van Den Heever, Cape Town, South Africa). Reprinted by permission from Springer: Springer Nature, Magnetic resonance imaging of the skeletal musculature, MRI of muscle injuries, Simon Dimmick, Christoph Rehnitz, Marc-André Weber, and James M. Linklater, 2014

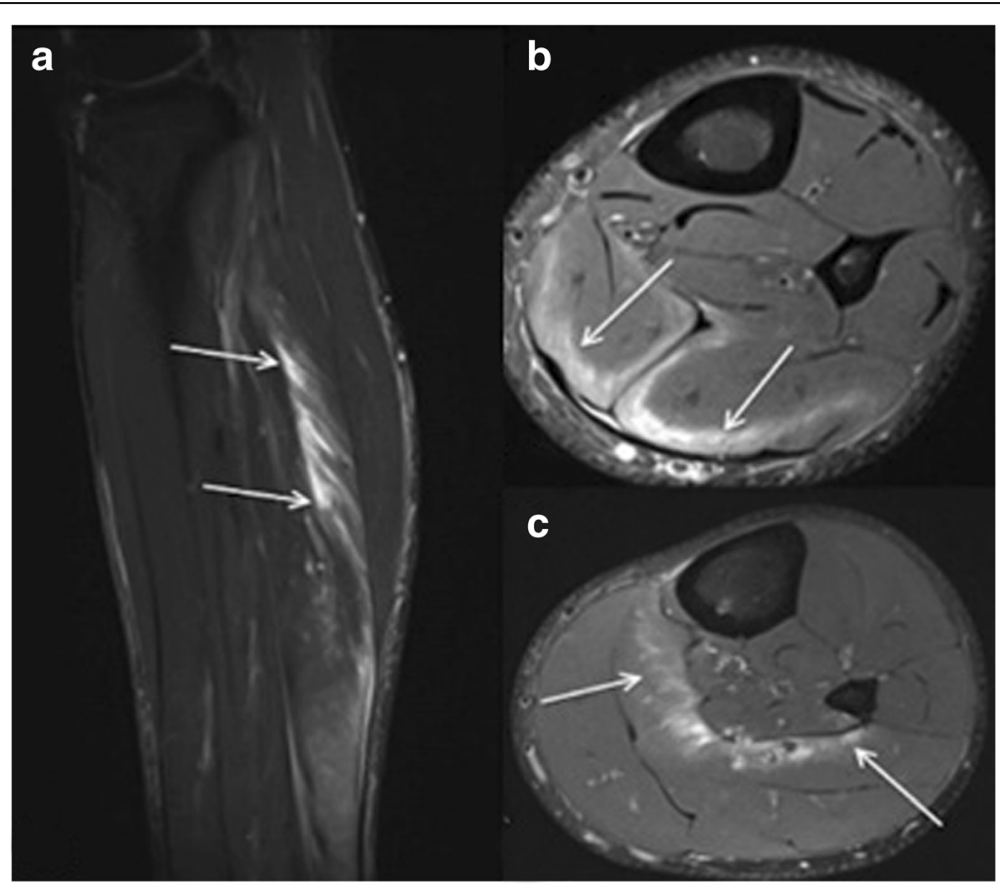

Fig. 8 Sagittal STIR (a) and axial proton density-weighted MR images with fat saturation (b, c) showing extensive post-exercise edema within the soleus muscle in an Australian Rules football player. The edema is centered around the myotendinous junctions, demonstrating a geographically emarginated distribution (arrows). The patient played an entire game 2 days after this MRI. Reprinted by permission from Springer: Springer Nature, Magnetic resonance imaging of the skeletal musculature, MRI of muscle injuries, Simon Dimmick, Christoph Rehnitz, Marc-André Weber, and James M. Linklater, 2014 


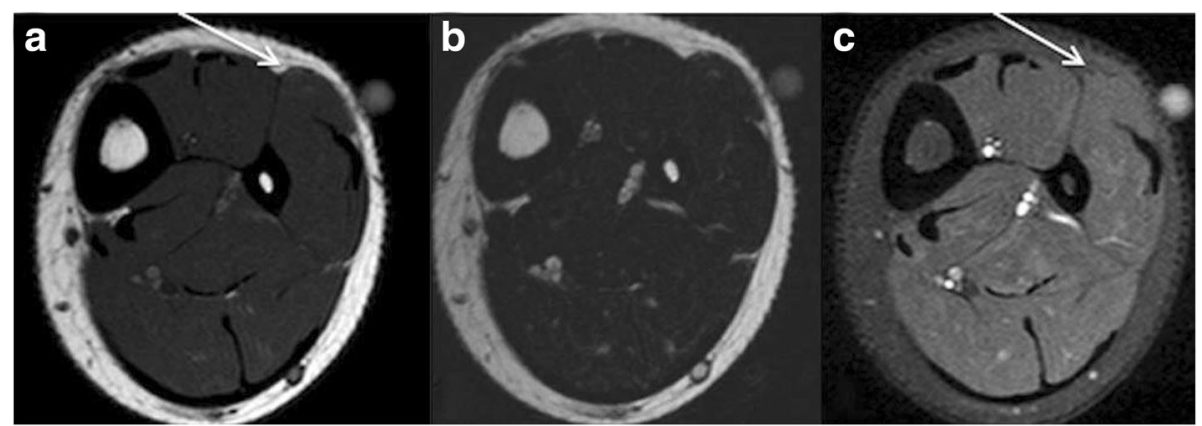

Fig. 9 A 16-year-old kickboxer with a suspected "tumor" of the lateral leg, location marked with a capsule. Axial T1 (a), T2 (b), and post contrast T1f-weighted (c) MR images demonstrate a muscle hernia of the peroneus longus muscle with outpouching of the muscle through the antero-lateral investing fascia. The hernia follows the imaging characteristics of the muscle tissue in all sequences. There was a history of direct trauma while playing soccer 1 year ago, presumably resulting in a tear of the investing fascia. The patient noted an increase in size during training/muscle activity. Reprinted by permission from Springer: Springer Nature, Magnetic resonance imaging of the skeletal musculature, MRI of muscle injuries, Simon Dimmick, Christoph Rehnitz, Marc-André Weber, and James M. Linklater, 2014

\section{Research on muscle injuries}

MR techniques that are used as research tools in the assessment of the skeletal muscles are blood oxygenation level-dependent (BOLD), diffusion tensor imaging (DTI), T2 mapping, and intravoxel incoherent motion (IVIM) [14, 23, 30, 31]. These techniques allow for non-invasive functional assessment of peripheral microvasculature in the skeletal muscles. While these techniques have currently no role in clinical routine, they improve the understanding of muscular and vascular physiology and alterations of microcirculation.

At BOLD MRI, which is founded on the different magnetic properties of oxy- and deoxyhemoglobin, blood is used as an endogenous contrast agent. BOLD allows to depict micro-vascularization, metabolism, and vascular insufficiency as in compartment syndrome $[7,14,32]$. In skeletal muscles, the BOLD contrast is generated by the microcirculation yielding that is very sensitive for alterations of the physiological oxygen supply and demand [7].
DTI of the muscle tissue allows examining the microarchitecture, the extraction of diffusion indices, and fiber tracking. DTI assesses integrity and orientation of muscle fibers. Changes at microscopic level like $z$-band disruptions can be detected. To monitor the conditions of the skeletal muscle, DTI is evolving to a clinical feasible tool and it may help to understand the pathophysiology in chronic exertional compartment syndrome [30].

T2 mapping detects muscle activation in specific groups and early fatty atrophy. During and after activity, T2 increases and the recruitment and capacity can be determined. T2 mapping may detect early microscopic fatty atrophy, not detected with morphologic MRI, represented as increased T2 values [14,32]. Other advanced MR imaging techniques for muscle injuries are MR spectroscopy measuring the muscle energy and lipid metabolism and ASL detecting the blood flow within the muscle tissue.

IVIM extracts information regarding microvascular blood flow out of multiple $b$ value diffusion acquisitions.

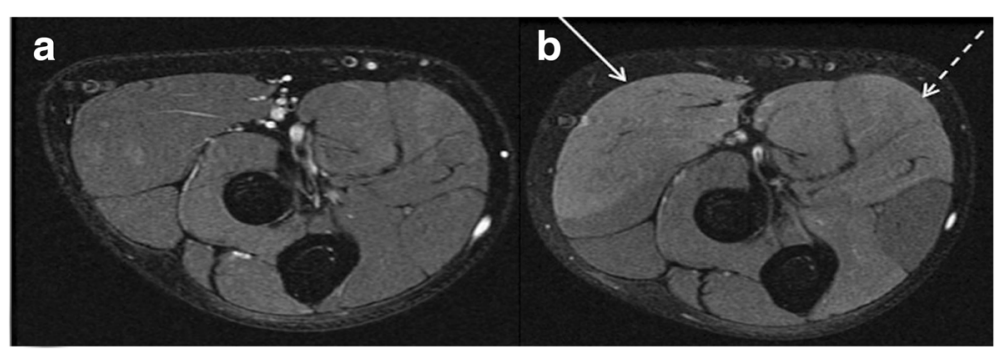

Fig. 10 Axial proton density-weighted MR image with fat saturation. Exercise-induced compartment syndrome, (a) forearm prior to exercise, (b) post-exercise swelling and ground glass-like edema within the brachioradialis, and humeral head of pronator teres muscles (arrow), while the remainder of the flexor pronator muscle group appears normal, with the exception of flexor carpi ulnaris (dashed arrows). Reprinted by permission from Springer: Springer Nature, Magnetic resonance imaging of the skeletal musculature, MRI of muscle injuries, Simon Dimmick, Christoph Rehnitz, Marc-André Weber, and James M. Linklater, 2014 


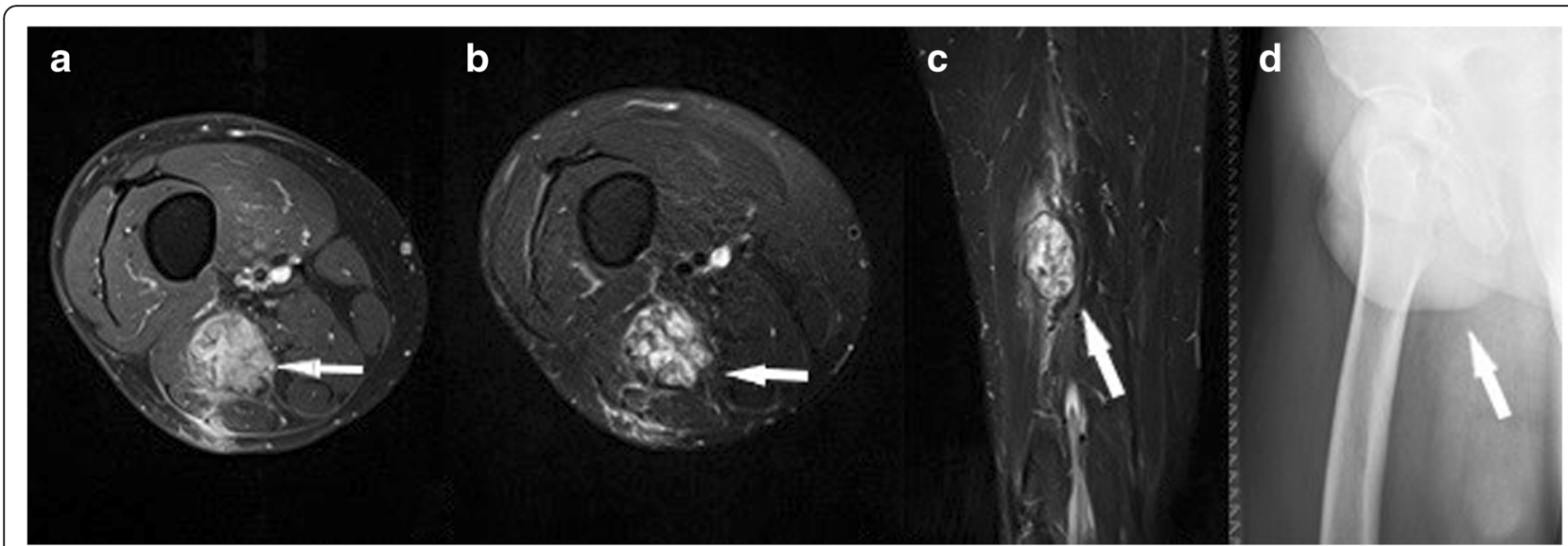

Fig. 11 Axial T1fs with contrast medium (a), axial T2fs (b), coronal STIR (c), and standard radiograph (d), depicting myositis ossificans in the hamstrings

It can determine an increase in microvascular perfusion in a specific segment after a particular task and can correlate this perfusion with the duration of the effort. Also, this method has shown to be able to quantify microvascularity and microstructure, evaluating the depletion of the capillaries and the degradation of myofibers, thus useful in the evaluation of dermatomyositis [23, 31].

\section{Conclusion}

The reference standard of imaging muscle injuries of the hip and thigh is MRI using fluid-sensitive and T1-weighted sequences. Typical findings are edema, hematoma, and partial or complete muscles tears. Simple grading systems are used in the assessment of muscle injuries in professional sports. However, specific imaging features such longitudinal length and volume of the muscle tear and involvement of the intramuscular component of the tendon have recently shown a significant impact on prognosis. These features should be precisely described, and a close collaboration with the team doctor is very helpful.

In order to optimally support athletes and team doctors, radiologists should be aware of the most common differential diagnoses delayed onset muscle soreness and post-exercise edema and complications like heterotopic ossification, compartment syndrome, or muscle herniation.

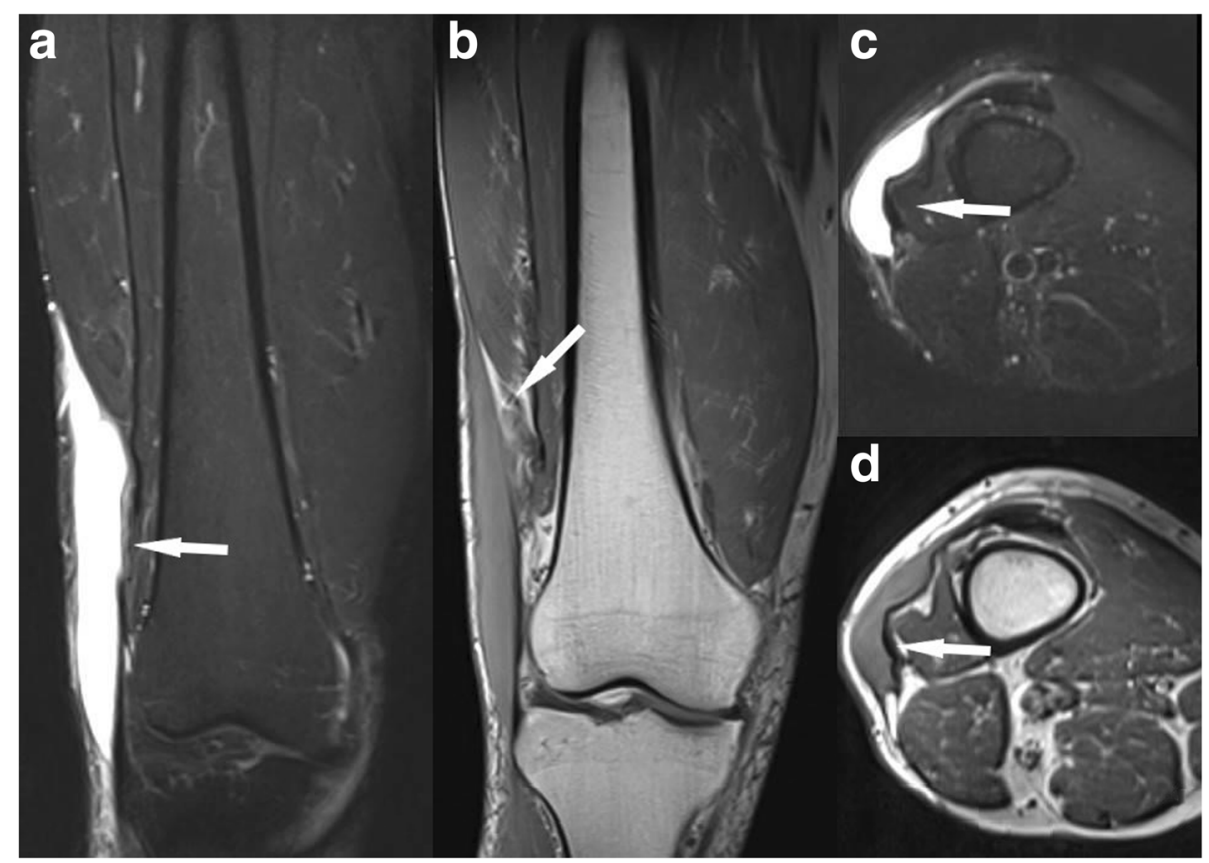

Fig. 12 Coronal PDfs (a) and T1 (b), axial PDfs (c), and T1 (d). Morel-Lavallée lesion of the distal thigh 


\section{Authors' contributions}

All authors read and approved the final manuscript.

\section{Competing interests}

The authors declare that they have no competing interests.

\section{Publisher's Note}

Springer Nature remains neutral with regard to jurisdictional claims in published maps and institutional affiliations.

\section{Author details}

'Institute of Diagnostic and Interventional Radiology, Pediatric Radiology and Neuroradiology, University Medical Center Rostock, Ernst-Heydemann-Str. 6, 18057 Rostock, Germany. ${ }^{2}$ Department of Radiology and Medical Imaging, University Emergency Hospital Bucharest, Bucharest, Romania. ${ }^{3}$ Department of Radiology, University Hospital, LMU Munich, Munich, Germany. ${ }^{4}$ Diagnostic and Interventional Radiology, Heidelberg University Hospital, Heidelberg, Germany.

Received: 24 July 2018 Accepted: 25 October 2018

Published online: 15 February 2019

\section{References}

1. Guermazi A, Roemer FW, Robinson P, Tol JL, Regatte RR, Crema MD (2017) Imaging of muscle injuries in sports medicine: sports imaging series. Radiology 285(3):1063

2. Robinson P, White LM (2005) The biomechanics and imaging of soccer injuries. Semin Musculoskelet Radiol 9(4):397-420

3. Ekstrand J, Askling C, Magnusson H, Mithoefer K (2013) Return to play after thigh muscle injury in elite football players: implementation and validation of the Munich muscle injury classification. Br J Sports Med 47(12):769-774

4. Mueller-Wohlfahrt HW, Haensel L, Mithoefer K et al (2013) Terminology and classification of muscle injuries in sport: the Munich consensus statement. Br J Sports Med 47(6):342-350

5. Ekstrand J, Hägglund M, Waldén M (2011) Epidemiology of muscle injuries in professional football (soccer). Am J Sports Med 39(6):1226-1232

6. Ekstrand J, Healy JC, Waldén M, Lee JC, English B, Hägglund M (2012) Hamstring muscle injuries in professional football: the correlation of MRI findings with return to play. Br J Sports Med 46(2):112-117

7. Dimmick S, Rehnitz C, Weber MA, Linklater JM (2014) MRI of muscle injuries. In: Weber MA (editor) Magnetic Resonance Imaging of the Skeletal Musculature. 2014, Springer-Verlag Berlin Heidelb. p. 187-219

8. Guermazi A, Roemer FW, Crema MD (eds) (2016) Imaging in sports-specific MSK injuries. Springer International Publishing

9. Ekstrand J, Hägglund M, Waldén M (2011) Injury incidence and injury patterns in professional football: the UEFA injury study. Br J Sports Med 45(7):553-558

10. Lückerath C, Rehnitz C (2017) Muscle injuries in athletes: the value of magnetic resonance imaging. Radiologe 57(12):1012-1018

11. Järvinen TA, Järvinen $T L$, Kääriäinen $M$, Kalimo $H$, Järvinen $M(2005)$ Muscle injuries: biology and treatment. Am J Sports Med 33(5):745-764

12. Palmer WE, Kuong SJ, Elmadbouh HM (1999) MR imaging of myotendinous strain. AJR Am J Roentgenol 173(3):703-709

13. Mason DL, Dickens VA, Vail A (2012) Rehabilitation for hamstring injuries. Cochrane Database Syst Rev 12:Cd004575

14. Crema MD, Yamada AF, Guermazi A, Roemer FW, Skaf AY (2015) Imaging techniques for muscle injury in sports medicine and clinical relevance. Curr Rev Musculoskelet Med 8(2):154-161

15. Hayashi D, Hamilton B, Guermazi A, de Villiers R, Crema MD, Roemer FW (2012) Traumatic injuries of thigh and calf muscles in athletes: role and clinical relevance of MR imaging and ultrasound. Insights Imaging 3(6):591-601

16. Rybak LD, Torriani M (2003) Magnetic resonance imaging of sports-related muscle injuries. Top Magn Reson Imaging 14(2):209-219

17. Nozaki T, Tasaki A, Horiuchi S et al (2016) Predicting retear after repair of full-thickness rotator cuff tear: two-point Dixon MR imaging quantification of fatty muscle degeneration-initial experience with 1 year follow-up. Radiology 280(2):500-509

18. Nozaki T, Tasaki A, Horiuchi S et al (2015) Quantification of fatty degeneration within the supraspinatus muscle by using a 2-point Dixon method on 3-T MRI. AJR Am J Roentgenol 205(1):116-122
19. Connell DA, Schneider-Kolsky ME, Hoving $\mathrm{J}$ et al (2004) Longitudinal study comparing sonographic and MRI assessments of acute and healing hamstring injuries. AJR Am J Roentgenol 183(4):975-984

20. Linklater JM, Hamilton B, Carmichael J, Orchard J, Wood DG (2010) Hamstring injuries: anatomy, imaging, and intervention. Semin Musculoskelet Radiol 14(2):131-161

21. Hallén A, Ekstrand J (2014) Return to play following muscle injuries in professional footballers. J Sports Sci 32(13):1229-1236

22. Blankenbaker DG, Tuite MJ (2010) Temporal changes of muscle injury. Semin Musculoskelet Radiol 14(2):176-193

23. Nguyen A, Ledoux JB, Omoumi P, Becce F, Forget J, Federau C (2016) Application of intravoxel incoherent motion perfusion imaging to shoulder muscles after a lift-off test of varying duration. NMR Biomed 29(1):66-73

24. Counsel P, Breidahl W (2010) Muscle injuries of the lower leg. Semin Musculoskelet Radiol 14(2):162-175

25. Koh ES, McNally EG (2007) Ultrasound of skeletal muscle injury. Semin Musculoskelet Radiol 11(2):162-173

26. Bates DG (2001) Dynamic ultrasound findings of bilateral anterior tibialis muscle herniation in a pediatric patient. Pediatr Radiol 31(10):753-755

27. Nguyen JT, Nguyen JL, Wheatley MJ, Nguyen TA (2013) Muscle hernias of the leg: a case report and comprehensive review of the literature. Can J Plast Surg 21(4):243-247

28. Sharma N, Kumar N, Verma R, Jhobta A (2017) Tibialis anterior muscle hernia: a case of chronic, dull pain and swelling in leg diagnosed by dynamic ultrasonography. Pol J Radiol 82:293-295

29. Tejwani SG, Cohen SB, Bradley JP (2007) Management of Morel-Lavallee lesion of the knee: twenty-seven cases in the national football league. Am J Sports Med 35(7):1162-1167

30. Noseworthy MD, Davis AD, Elzibak AH (2010) Advanced MR imaging techniques for skeletal muscle evaluation. Semin Musculoskelet Radio 14(2):257-268

31. Sigmund EE, Baete SH, Luo T et al (2018) MRI assessment of the thigh musculature in dermatomyositis and healthy subjects using diffusion tensor imaging, intravoxel incoherent motion and dynamic DTI. Eur Radiol 28(12): 5304-5315

32. Varghese J, Scandling D, Joshi R et al (2015) Rapid assessment of quantitative $\mathrm{T} 1, \mathrm{~T} 2$ and $\mathrm{T} 2 *$ in lower extremity muscles in response to maximal treadmill exercise. NMR Biomed 28(8):998-1008

\section{Submit your manuscript to a SpringerOpen ${ }^{\circ}$ journal and benefit from:}

- Convenient online submission

- Rigorous peer review

- Open access: articles freely available online

High visibility within the field

- Retaining the copyright to your article

Submit your next manuscript at $>$ springeropen.com 